KfK 4032

Januar 1986

\title{
Coulomb Dissociation as a Source of Information on Radiative Capture Processes of Astrophysical Interest
}

\author{
G. Baur, C. A. Bertulani, H. Rebel \\ Institut für Kernphysik
}

\section{Kernforschungszentrum Karlsruhe}




\title{
KERNFORSCHUNGSZENTRUM KARLSRUHE
}

Institut für Kernphysik

KEK 4032

\begin{abstract}
COULOMB DISSOCIATION AS A SOURCE
OF INFORMATION ON RADIATIVE CAPTURE PROCESSES

OF ASTROPHYSICAL INTEREST
\end{abstract}

G. Baur ${ }^{+}$, C.A. Bertulani ${ }^{++}$and H. Rebel

+ IKP der Kernforschungsanlage Jülich

${ }^{++}$on leave from Federal University of Rio de Janeiro, Brazil

Kernforschungszentrum Karlsruhe $\mathrm{GmbH}$, Karlsruhe 
Als Manuskript vervielfältigt

Für diesen Bericht behalten wir uns alle Rechte vor

Kernforschungszentrum Karlsruhe $\mathrm{GmbH}$ Postfach 3640, 7500 Karlsruhe 1

ISSN 0303-4003 
Abstract

Experimental studies of the break up of light nuclear projectiles in the coulomb field of a heavy nucleus, acting as a source of virtual photons, are proposed as an access to information about the reverse reaction, the fusion of the fragment particles at small relative energies. The mechanism of coulomb dissociation is studied and the cross section of such reactions, being potentially of astrophysical interest, is estimated. The conditions of dedicated experimental investigations are discussed.

COULOMB DISSOZIATION ALS INFORMATIONSQUELLE ÜBER STRAHLUNGSEINFANGPROZESSE VON ASTROPHYSIKALISCHEM INTERESSE

Zusammenfassung

Es werden experimentelle Studien des Aufbruchs leichter Ionen im Coulombfeld schwerer Kerne, die beim Vorbeiflug als virtuelle Photonenquelle wirken, vorgeschlagen als ein möglicher Zugang $\mathrm{zu}$ Informationen über die Umkehrreaktion, der Fusion leichter nuklearer Teilchen bei kleinen Relativenergien. Der Mechanismus der CoulombDissoziation wird untersucht, und die Wirkungsquerschnitte von Reaktionen von speziellem astrophysikalischen Interesse werden abgeschätzt. Die Bedingungen gezielter experimenteller Untersuchungen werden diskutiert. 
Contents

Page

1. Introduction 1

2. Virtual photon spectrum and double-differential cross section for coulomb dissociation

3. Application to specific examples and cross section estimates

4. Conditions for experimental investigations

6. Conclusions 
1. Introduction

The cross sections for radiative capture of $\alpha$-particles, deuterons and protons by light nuclei at very low relative energies are of particular importance for the understanding of the nucleosynthesis of chemical elements and for determining the relative elemental abundances in stellar burning processes at various astrophysical sites ${ }^{1,2}$. However, the direct experimental determination of the cross sections at astrophysically relevant energies under laboratory conditions is rather difficult or even precluded, mainly as the Coulomb barrier strongly suppresses the cross sections for the reactions of interest. For example, the ${ }^{3} \mathrm{He}\left({ }^{4} \mathrm{He}, \gamma\right)^{7}$ Be reaction, which at solar temperatures affects the solar neutrino flux and bears strongly on the solar neutrino problem $^{3}, 4$, is experimentally studied 4,5 down to $\mathrm{CM}$-energies $\mathrm{E}_{\mathrm{CM}}=165 \mathrm{keV}$, while the cross section is actually needed at $\mathrm{E}_{\mathrm{CM}}=1-20 \mathrm{keV}$. A similar situation is found for the ${ }^{12} \mathrm{C}(\alpha, \gamma){ }^{16} \mathrm{O}$ reaction ${ }^{6}$, which is important for the stellar helium-burning process and where the values of the low-energy cross section (at $\mathrm{E}_{\mathrm{CM}} \approx 0.3 \mathrm{MeV}$ corresponding to temperatures of $2 \times 10^{8} \mathrm{~K}$ ) are actually a matter of controversial discussion presently. In cases of nonresonant direct capture reactions the energy dependence is dominated by the coulomb barrier penetration, which is usually factored out by defining the astrophysical s-factor

$$
S\left(E_{C M}\right)=\sigma_{\text {Capt }} \cdot E_{C M} \exp (2 \pi \eta)
$$

where

$$
\eta=\frac{z_{1} z_{2} e^{2}}{n v}
$$

is the usual coulomb parameter. This S-factor shows a smooth energy dependence and seems to be adequate for an extrapolation of the measured values to astrophysically relevant energy ranges. But in most cases of interest the extrapolation covers several orders of magnitude and is particularly suspect if resonances and subthreshold resonances are expected to be present in the considered reaction (see ref. 2). In addition, the extrapolation needs often considerable theoretical support and bias, and despite of strong efforts to understand nuclear 
reactions on theoretical grounds, it appears to be generally impossible to predict the astrophysically interesting cross sections with sufficient accuracy.

In view of this situation, all dedicated efforts which are able to explore additional experimental information on the quantities determining low-energy nuclear reaction cross sections are of considerable interest. Recently the investigation of continuum stripping processes has been discussed ${ }^{7}$ as a possible method to overcome the problem arising from the coulomb barrier. However, the method involves a theoretical reaction model which might cast some doubts on the results.

In the present study we analyse a different approach which has been recently proposed ${ }^{8}$ for the investigation of electromagnetic transitions between a bound state of two nuclear particles and continuum states at small relative energies. The proposal suggests to use the nuclear coulomb field as a source of the photodisintegration processes. In fact, instead of studying directly the radiative capture process

$$
b+c \rightarrow a+\gamma
$$

one may consider the time reversed process (a being in the ground state)

$$
r+a \rightarrow b+c
$$

The corresponding cross sections are related by the detailed balance theorem

$$
\sigma(b+c \rightarrow a+\gamma)=\frac{\left(2 j_{a}+1\right) \cdot 2}{\left(2 j_{b}+1\right)\left(2 j_{c}+1\right)} \frac{k_{\gamma}^{2}}{k^{2}} \sigma(a+\gamma+b+c)
$$

The wave number in the $(b+c)$-channel is

$$
\mathrm{k}^{2}=\frac{2 \mu_{\mathrm{bc}}{ }^{\mathrm{E}} \mathrm{CM}}{\mathrm{h}^{2}}
$$

with $\mu_{\mathrm{bc}}$ the reduced mass while the photon wave number is given

$$
k_{\gamma}=\frac{E_{\gamma}}{h c}=\frac{E_{C M}+Q}{\hbar c}
$$


(neglecting a small recoil correction) in terms of the Q-value of the capture reaction (eq. 1.2). Except for the extreme case very close to the threshold $(k \rightarrow 0)$, we have $k_{\gamma} \ll k$, so that the phase space favours the photointegration cross section as compared to the radiative capture. However, direct measurements of the photodisintegration near the break up threshold do hardly provide experimental advantages and seem presently impxacticable (see ref. 8). On the other hand the copious source of virtual photons ${ }^{9}$ acting on a fast charged nuclear projectile when passing the coulomb field of a (large $z$ ) nucleus offers a more promising way to study the photodisintegration process as coulomb dissociation. Fig. 1 indicates schematically the dissociation reaction.

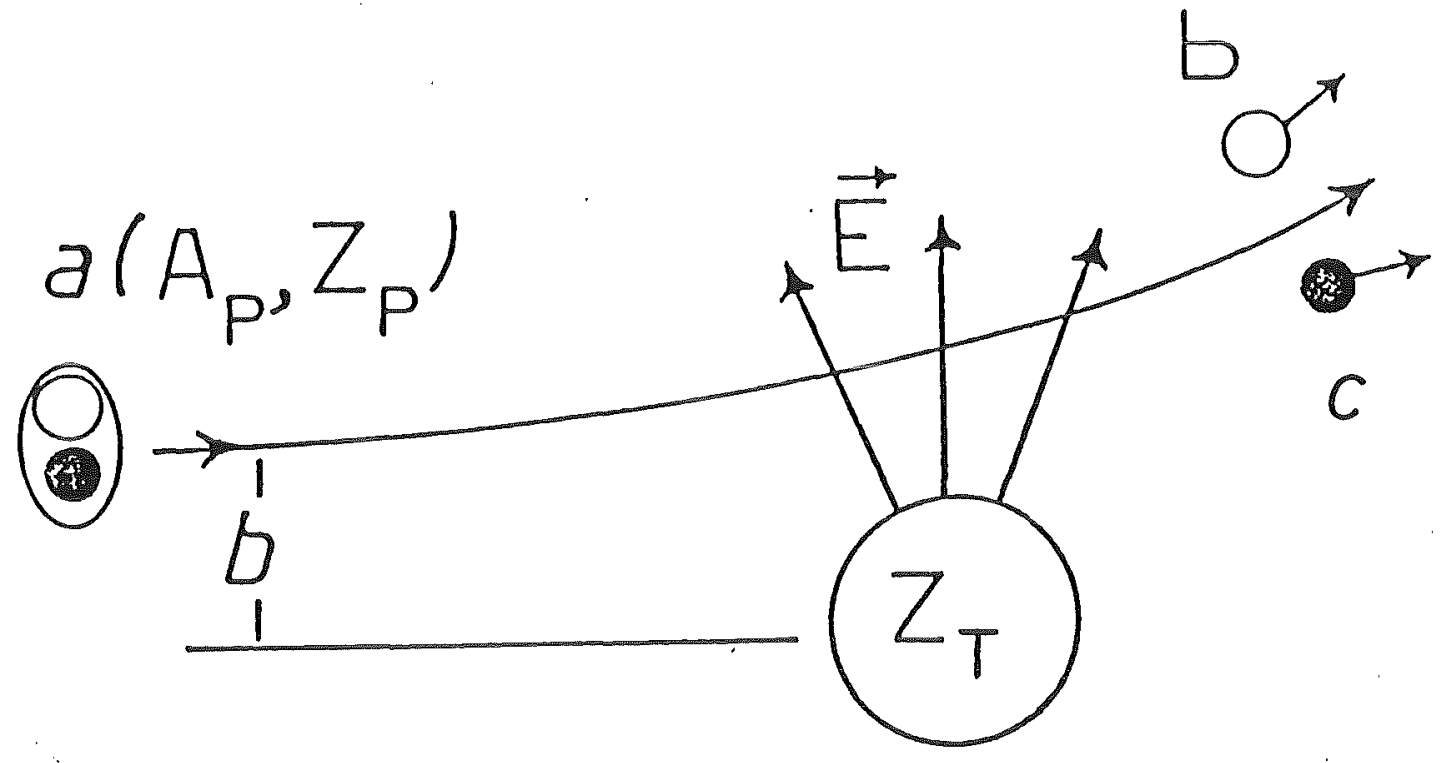

Fig. 1 Coulomb dissociation $a \rightarrow b+c$ in the field of a target nucleus $\left(z_{T}\right)$

At a sufficiently high projectile energy the two fragments $b$ and $c$ emerge with rather high energies (around the beam-velocity energies) which facilitates the detection of these particles. At the same time the choice of adequate kinematical conditions for coincidence measurements allows to study rather low relative energies of $\mathrm{b}$ and $\mathrm{c}$ and to ensure that the target nucleus stays in the ground state (elastic break up). In addi- 
tion, it turns out that the large number of virtual photons seen by the passing projectile leads to an enhancement of the cross section. In the following we give first some theoretical estimates of the Coulomb dissociation cross section on the basis of sufficiently accurate theoretical descriptions ${ }^{9-12}$. We apply the results to two examples of actual astrophysical interest and consider briefly the experimental conditions and the feasibility of such coulomb dissociation experiments.

2. Virtual photon spectrum and double-differential cross section for coulomb dissociation

The calculation of the double differential cross section for a deflection of a charged projectile by the angle $\theta$ with subsequent coulomb excitation irto the continuum to the excitation energy $E_{x}\left(\approx E_{\gamma}\right)$ starts with following assumptions

(i) The influence of the strong nuclear field on the projectile motion and the excitation process can be neglected. This is presumably the case for sufficiently large impact parameters b (see Fig. 1) i.e. small scattering angles.

(ii) The application of a first order theory is expected to be of sufficient accuracy, thus disregarding "post-acceleration" effects of the broken up particles $b$ and $c$ in the nuclear Coulomb field, which might disturb the extraction of the correct energy $\mathrm{E}_{\mathrm{bc}}$ of the relative motion. Such second order effects must be incorporated in a future theory.

The double differential cross section for Coulomb excitation of the projectile to a state of electric multipole order $\lambda$ can be expressed in terms of the reduced transition probabilities $B(E \lambda)$ of the corresponding transition, in a first order perturbation theory by 


$$
\frac{d^{2} \sigma}{d \Omega d E_{\gamma}}=\left(\frac{Z_{T} e}{\hbar v}\right)^{2} \quad a^{-2 \lambda+2} d f_{E \lambda}(\theta, \xi) \quad B\left(E \lambda, I_{i} \rightarrow I_{E}\right) \rho_{f}\left(E_{\gamma}\right)
$$

where $v$ is the relative velocity, $z_{T}$ is the target charge, a is half the distance of closest approach in a head-on collision, $\rho_{f}\left(E_{\gamma}\right)$ is the density of final states of the projectile per energy interval, and the adiabaticity parameter $\xi=\frac{E_{\gamma} a}{-\frac{K}{V}}=\frac{\omega a}{v}$. The $B(E \lambda)$ value is defined as in ref. 13 and it has the ${ }^{-1 / v}$ units $e^{2} \mathrm{fm}^{2 \lambda}$. The function $d f_{E \lambda}(\theta, \xi)$ (see Ref. 13) can be calculated in different ways: (i) in an exact quantum mechanical way using coulomb wave functions for the incident and outgoing particle, (ii) by a semiclassical method where the projectile moves on a Rutherford orbit and is excited by the time-dependent electric field of the target. For the cases which will be of interest here, the first procedure which is computationally more complex, is expected to provide similar results as the second method.

The $B(E \lambda)$ value is related to the photoabsorption cross section $\sigma_{E \lambda}$ photo by

$$
\sigma_{E \lambda}^{\text {photo }}=\frac{(2 \pi)^{3}(\lambda+1)}{\lambda[(2 \lambda+1) ! !]^{2}}\left(k_{Y}\right)^{2 \lambda-1} B\left(E \lambda, I_{i} \rightarrow I_{E}\right) \rho_{f}\left(E_{Y}\right)
$$

and we can rewrite eq. $(2.1)$ as

$$
\frac{d^{2} \sigma}{d \Omega d E_{\gamma}}=\frac{1}{E_{\gamma}} \frac{d n_{E \lambda}}{d \Omega} \sigma_{E \lambda}^{\text {photo }}
$$

where

$$
\frac{d n_{E \lambda}}{d \Omega}=z_{T}^{2} \alpha \frac{\lambda[2 \lambda+1) ! !]^{2}}{(2 \pi)^{3}(\lambda+1)} \xi^{-2 \lambda+2} \quad\left(\frac{c}{v}\right)^{2 \lambda} d f_{E \lambda}(\Theta, \xi)
$$

with $\alpha=\frac{e^{2}}{\hbar c} \cong \frac{1}{137}$. The function $\frac{d n_{E} \lambda}{d \Omega}$ does not depend on the internal structure of the projectile. It only depends on the kinematics of the relative motion and on the excitation energy $E_{Y}=\hbar \omega$. 
We $\operatorname{call} \frac{\mathrm{dn}_{E \lambda}}{\mathrm{d} \Omega}$ the virtual photon number per unit solid angle. The first calculations of the function $d f_{E \lambda}(\theta, \xi)$ was performed by TerMartirosyan $^{14}$. We restrict ourselves to the most important case, $\lambda=1$, for which $\mathrm{df}_{\mathrm{E} 1}(\theta, \xi)$ can be expressed in terms of the modified Bessel functions $K_{V}(x)$. Inserting that expression in eq. (2.4) we obtain

$\left.\frac{d n_{E 1}}{d \Omega}=\frac{z_{T}^{2} \alpha}{4 \pi^{2}}\left(\frac{c}{v}\right)^{2} \varepsilon^{4} \xi^{2} e^{-\pi \xi}\left(\frac{\varepsilon^{2}-1}{\varepsilon^{2}}\right)\left[K_{i \xi}(\varepsilon \xi)\right]^{2}+\left[K_{i \xi}^{\prime}(\varepsilon \xi)\right]^{2}\right)$

where the excentricity parameter $\varepsilon=1 / \sin (\theta / 2)$ and $K_{i \xi}^{\prime}(x)$ means the derivative of $K_{i \xi}(x)$ with respect to the argument.

For relativistic projectile energies the Rutherford trajectory can be substituted by a straight-line and instead of the scattering angle $\theta$ the concept of impact parameter $b$ is used. The virtual photon method in that case was first introduced by Enrico Fermi ${ }^{15}$ and later developed by Weizsäcker and Williams ${ }^{16}$ (see also Ref. 17). It is given by

$$
\frac{d n_{E 1}}{2 \pi b d b}=\frac{z_{T^{\alpha}}^{2}}{16 \pi^{2}}\left(\frac{\omega}{\gamma v}\right)^{2}\left(\frac{c}{V}\right)^{2}\left[K_{1}^{2}(x)+\frac{1}{\gamma^{2}} K_{O}^{2}(x)\right.
$$

where $\gamma$ is the relativistic factor $\gamma=\left(1-\frac{v^{2}}{c^{2}}\right)^{-1 / 2}$ and $x=\frac{\omega b}{\gamma v}$. Since for a Rutherford trajectory the impact parameter is related to the scattered angle by the relation $b=a \operatorname{ctg}\left(\frac{\Theta}{2}\right)$ we can rewrite eq. $(2.6)$ as

$$
\left(\frac{\mathrm{dn} E 1}{\mathrm{~d} \Omega}\right)_{\text {relat. }}=\frac{\mathrm{z}_{\mathrm{T}}^{2}}{4 \pi^{2}} \xi^{2} \varepsilon^{4}\left(\frac{\mathrm{c}}{\gamma \mathrm{V}}\right)^{2}\left[\mathrm{~K}_{1}^{2}(\mathrm{x})+\frac{1}{\gamma^{2}} \mathrm{~K}_{\mathrm{O}}^{2}(\mathrm{x})\right]
$$

of course, for relativistic energies $\theta \ll 1$ and $x=\frac{\varepsilon \xi}{\gamma} \cos \left(\frac{\Theta}{2}\right) \cong \frac{\varepsilon \xi}{\gamma}$.

For the nonrelativistic limit a small scattering angle is related to a large impact parameter trajectory $\left(\varepsilon \cong \frac{b}{a} \gg 1\right)$. If we assume $\xi \ll 1$, then by use of $\mathrm{K}_{0}^{\prime}=-\mathrm{K}_{1}$ we obtain from (2.5) 


$$
\frac{d n_{E 1}}{d \Omega}=\frac{z_{T}^{2}}{4 \pi^{2}} \varepsilon^{2}\left(\frac{c}{v}\right)^{2} x^{2}\left[k_{0}^{2}(x)+k_{1}^{2}(x)\right]
$$

which is just the eq. (2.7) for $\gamma \cong 1$.

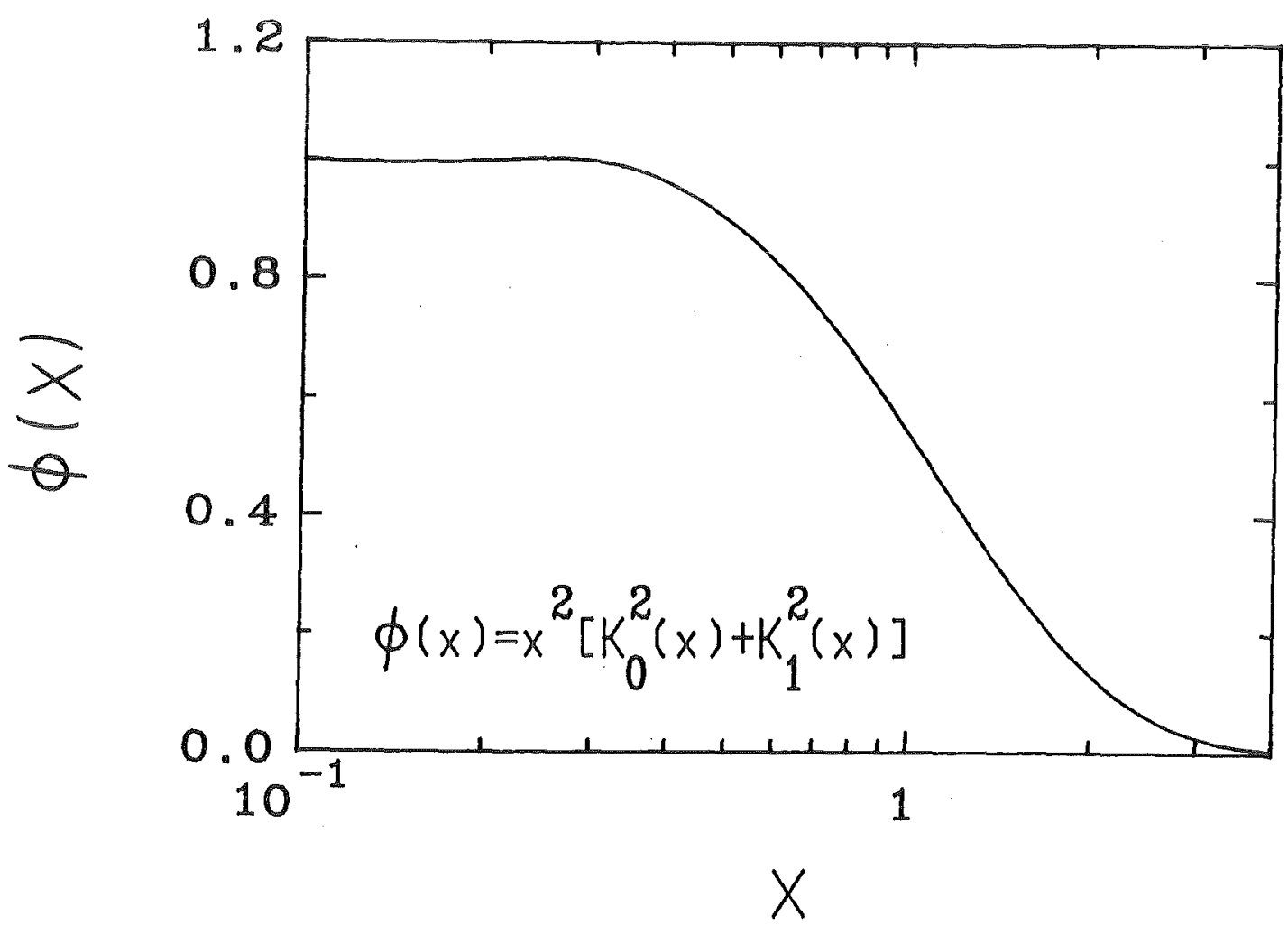

Fig. 2 The shape of the virtual photon spectrum as function of $x=\frac{\omega b}{\gamma v}$ (with $\left.E_{Y}=\hbar \omega\right)$ for a given impact parameter $b$

The shape of the virtual photon spectrum for a given impact parameter is seen in Fig. 2 where the adimensional function $\phi(x)=x^{2}\left[K_{0}^{2}(x)+k_{1}^{2}(x)\right]$ is plotted. In a crude approximation $\phi=1$ for $x \leq 1$, and $\phi=0$ for $x>1$. This means that the spectrum will contain all frequencies up to a maximum of order $\omega_{\max }$ $\cong \mathrm{V}$, and small impact parameter trajectories can lead to a great probability of exciting high-lying states of the projectile which preferentially decay by particle emission or disintegration.

For not too large impact parameters, which still lead to small scattering angles the Rutherford-bending of the trajectory 
is mainly reflected through the parameter $\xi$. In that case eq. (2.5) is, approximately,

$$
\frac{d n_{E 1}}{d \Omega}=\frac{z_{T}^{2} \alpha}{4 \pi^{2}} \varepsilon^{2}\left(\frac{c}{v}\right)^{2} e^{-\pi \xi} x^{2}\left\{\left[K_{i \xi}(x)\right]^{2}+K_{i \xi}^{\prime}(x)^{2}\right\}
$$

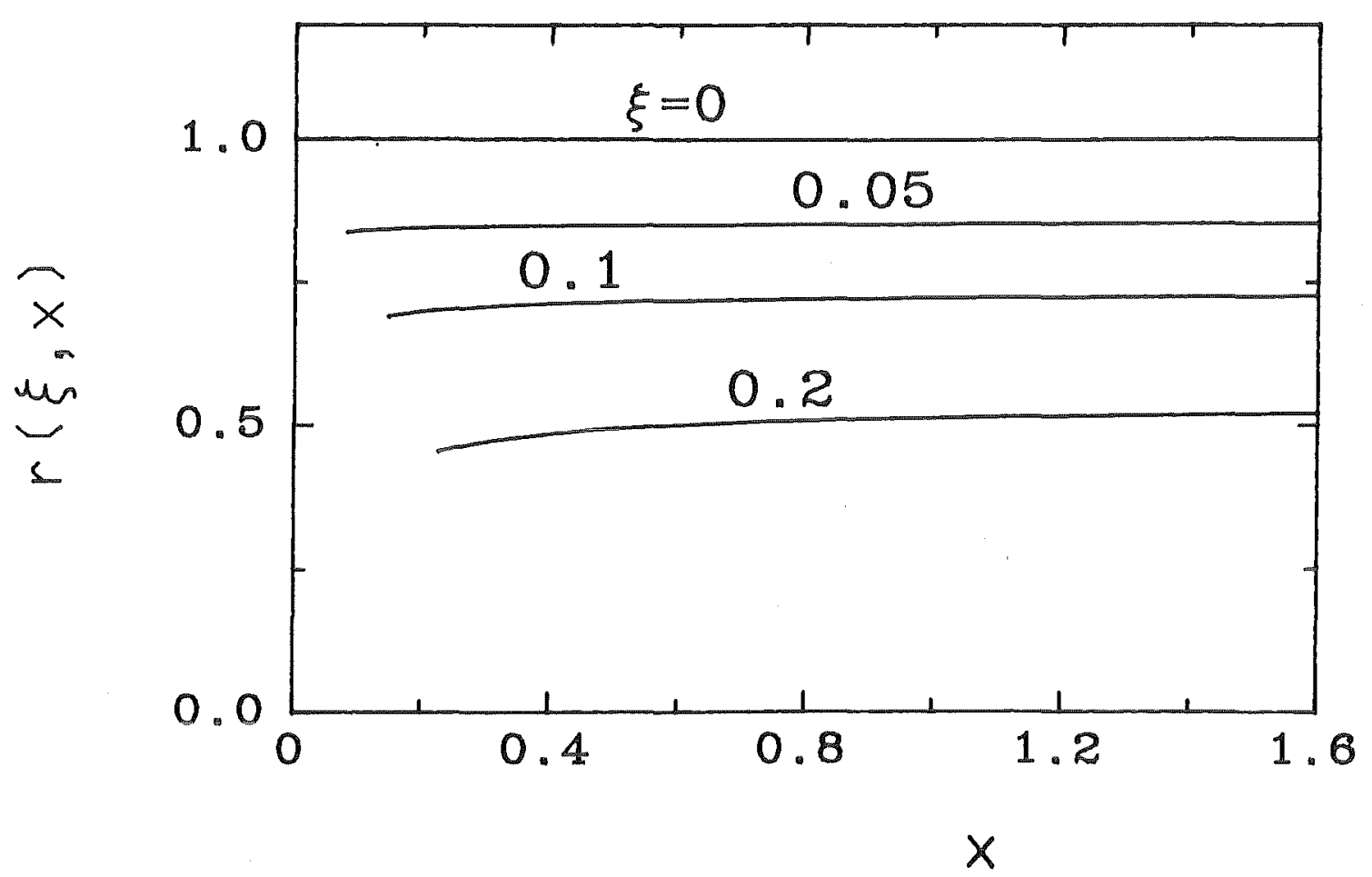

Fig. 3 Effect of Rutherford-bending of the projectile motion for different values of the adiabacity parameter $\xi=E_{\gamma} \cdot a /(\hbar v)$

Fig. 3 displays the ratio $r(\xi, x)=\frac{\text { eg. }(2.9)}{\text { eq. }(2.8)}$ which shows the effect of the Rutherford-bending to the straightline calculation. This effect increases steadily with $\xi$.

In eq. (2.5) the Rutherford trajectory is accounted for properly in the calculations, but retardation effects in the interaction are ignored. The reverse is true in the calculations which lead 
to eq. (2.6). While one can safely use eq. (2.5) in nonrelativistic problems and eq. (2.6) in relativistic ones, the previous discussion has shown that none of them is suitable for intermediate energy problems where both effects are present. But by a direct look at the eqs. (2.5) and (2.7) we see that the main effect of the Rutherford trajectory would be present in the imaginary indices

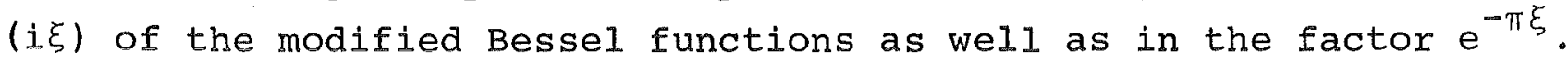
On the other hand retardation effects imply in the appearence of the $\gamma$-factors in the eq. (2.7), thus suggesting that one can account simultaneously for both effects by defining the new variable $\zeta=\frac{\xi}{\gamma}=\frac{\omega a}{\gamma v}$ and setting

$$
\begin{aligned}
\frac{d n_{E 1}}{d \Omega}=\frac{z_{T^{\alpha}}^{2}}{4 \pi^{2}} \zeta^{2} \varepsilon^{4} e^{-\pi \zeta}\left(\frac{c}{v}\right)^{2}\left\{\frac{1}{\gamma^{2}}\left(1-\frac{1}{\varepsilon^{2}}\right)\left[K_{i \zeta}(\varepsilon \zeta)\right]^{2}\right. & \\
+ & {\left.\left[K_{i \zeta}^{\prime}(\varepsilon \zeta)\right]^{2}\right\} }
\end{aligned}
$$

This equation reduces to the eq. (2.5) for $\gamma \cong 1$ and to eq. (2.7) for $\gamma \gg 1, \varepsilon \gg 1$ and should be a good improvement for the intermediate energy region.

According to eq. (2.3) the differential Coulomb excitation cross section integrated over angles is

$$
\frac{d \sigma}{d E_{\gamma}}=\frac{1}{E_{\gamma}} n_{E 1} \sigma_{E 1}^{\text {photo }}
$$

(with neglect of multipolarities $\lambda>1$ by assuming that the E1 contribution will be dominant). The virtual photon number $\mathrm{n}_{\mathrm{E} 1}$ is obtained by an integration of eq. (2.10) over all angles corresponding to pure coulomb trajectories. In terms of the excentricity parameter, this integral can be expressed as

$$
\begin{aligned}
\mathrm{n}_{E 1} & =\frac{2}{\pi} \mathrm{z}_{T^{2}}^{2} \zeta^{2} e^{-\pi \zeta}\left(\frac{c}{\mathrm{~V}}\right)^{2} \\
* & \int_{\varepsilon_{0}}^{\infty} \varepsilon d \varepsilon\left\{\frac{1}{\gamma^{2}}\left(1-\frac{1}{\varepsilon^{2}}\right)\left[\mathrm{K}_{i \zeta}(\varepsilon \zeta)\right]^{2}+\left[\mathrm{K}_{i \zeta}^{\prime}(\varepsilon \zeta)\right]^{2}\right\}
\end{aligned}
$$


The minimum value of the excentricity parameter depends on whether the relative motion energy is smaller or greater than the Coulomb barrier energy $\mathrm{E}_{\mathrm{B}}$ :

$$
\varepsilon_{0}=\int \frac{1 \text { for } E \leq E_{B}}{\sqrt{1+4\left(\frac{E}{E_{B}}\right)^{2}\left(1-\frac{E_{B}}{E}\right)}} \quad \text { for } E>E_{B}
$$

We see that when $E \gg E_{B}=\frac{Z_{P} Z_{T} e^{2}}{R}$, then $\varepsilon_{0} \cong \frac{2 E}{E_{B}}=\frac{R}{a}$, where $R$ is the sum of the two nuclear radii. The integration (2.12) can also be expressed in terms of the modified Bessel functions of imaginary or complex indices by means of the Lommel integral formulas ${ }^{18}$. This gives

$$
\begin{aligned}
n_{E 1}= & \frac{2}{\pi} z_{T}^{2} e^{-\pi \zeta}\left(\frac{c}{v}\right)^{2}\left(-x k_{i \zeta} k_{i \zeta}^{\prime}\right. \\
& -\frac{1}{2}\left(\frac{v}{c}\right)^{2} x^{2}\left[K_{i \zeta+1} K_{i \zeta-1}-K_{i \zeta}^{2}\right. \\
& \left.\left.+\frac{1}{\varepsilon}-\left\{K_{i \zeta}\left(\frac{\partial K_{\mu}^{\prime}}{\partial \mu}\right)_{\mu=i \zeta}-K_{i \zeta}^{\prime}\left(\frac{\partial K_{\mu}}{\partial \mu}\right)_{\mu=i \zeta}\right\}\right]\right)
\end{aligned}
$$

where all $\mathrm{K}^{\prime} \mathrm{s}$ are functions of $\mathrm{x}=\varepsilon_{\mathrm{o}} \zeta$. In the nonrelativistic limit $B=\frac{V}{C} \rightarrow 0, \varepsilon_{0} \rightarrow 1$ and we obtain

$$
\mathrm{n}_{\mathrm{E} 1}=-\frac{2}{\pi} \mathrm{z}_{\mathrm{T}}^{2} \alpha \xi \mathrm{e}^{-\pi \zeta}\left(\frac{\mathrm{C}}{\mathrm{V}}\right)^{2} \mathrm{~K}_{i \xi}(\xi) \mathrm{K}_{i \xi}^{\prime}(\xi)
$$

In the relativistic limit $B \rightarrow 1, \varepsilon_{0} \cong \frac{R}{a}+\infty$ and $\zeta=\frac{\xi}{\gamma} \rightarrow 0$, so that

$$
\mathrm{n}_{E 1}=\frac{2}{\pi} \mathrm{z}_{T^{\alpha}}^{2}\left(\frac{\mathrm{c}}{\mathrm{v}}\right)^{2}\left\{\mathrm{x} \mathrm{K}_{\mathrm{O}} \mathrm{K}_{1}-\frac{1}{2}\left(\frac{\mathrm{v}}{\mathrm{C}}\right)^{2} \mathrm{x}^{2}\left(\mathrm{~K}_{1}^{2}-\mathrm{K}_{\mathrm{O}}^{2}\right)\right\}
$$

where the $K^{\prime} s$ are functions of $x=\varepsilon_{0} \zeta \cong \frac{\omega R}{\gamma V}$

Of course, both expressions (2.15) and (2.16) agree with the known results of previous calculations (see e.g. Refs. 13 and 17). But, besides of reproducing the nonrelativistic and the relativistic limits, eq. (2.14) might be useful for intermediate energy problems. 
3. Application to specific examples and cross section estimates

We consider two specific reactions

(i) ${ }^{7} \mathrm{Be}+{ }^{208} \mathrm{~Pb} \rightarrow \alpha+{ }^{3} \mathrm{He}+{ }^{208} \mathrm{~Pb}-1.58 \mathrm{MeV}$

(ii) ${ }^{16} \mathrm{O}+{ }^{208} \mathrm{~Pb} \rightarrow{ }^{12} \mathrm{C}+\alpha+{ }^{208}{ }_{\mathrm{Pb}} \mathrm{g}-7.162 \mathrm{MeV}$

in which ${ }^{7} \mathrm{Be}$ and ${ }^{16} \mathrm{O}$ projectiles, respectively dissociate by the electromagnetic field experienced when passing a ${ }^{208} \mathrm{~Pb}$ nucleus with a sufficiently large impact parameter $b_{3}>R_{P b}+R_{p}$. After dissociation two fragments are emerging ( $\alpha-{ }^{3} \mathrm{He}$ or $12 \mathrm{C}^{\mathrm{p}}-\alpha$, respectively) and are detected (coincidently) in a geometry of small angular spacing so that small relative energies of the moving fragments are kinematically allowed.

The two cases are related to corresponding radiative capture reactions of actual astrophysical interest (see sect. 1). It has been found that in the ${ }^{12} \mathrm{C}(\alpha, \gamma){ }^{16} \mathrm{O}$ reaction the E2-component strongly competes with the isospin - forbidden E1 transition. Nevertheless we study here only the E1 contribution, which is dominating in the ${ }^{3} \mathrm{He}(\alpha, \gamma){ }^{7} \mathrm{Be}$ case, and we leave the extension to other multipolarities and E1-E2 interferences to more detailed studies. In principle, there appears no limitation to include higher electromagnetic multipole contributions.

The dependence of the virtual photon number $\mathrm{dn}_{E 1} / \mathrm{d} \Omega$ on the excitation energy $E_{\gamma}$, on the impact parameter $b$ and on the incident energy of the projectile $\left(A_{p}, z_{p}\right)$ can be expressed by eq. (2.8) as

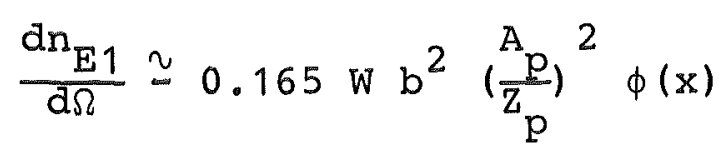

with $\mathrm{W}$ denoting the projectile energy per mass in units of $\mathrm{MeV} / \mathrm{amu}$ and $b$ given in units of $\mathrm{fm}$. The explicit dependence on $z_{T}$ apparentIy disappears since $\varepsilon \approx \mathrm{b} / \mathrm{a} \cong 2 \mathrm{bA} \mathrm{A}_{\mathrm{p}} \cdot \mathrm{W} /\left(\mathrm{z}_{\mathrm{p}} \mathrm{z}_{\mathrm{T}} \mathrm{e}^{2}\right)$. The virtual photon number per unit solid angle obviously increases linearly with the specific projectile energy $W$ and quadratically with the value of the impact parameter $b$ as long as $x=\omega b /(\gamma v)<<1$ so that $\phi(x) \approx 1$. Preparing optimum conditions for a given energy $w, x<<1$ has to be ensured by keeping $b<b_{1 i m}$ where $b_{1 i m}$ is determined by the adia- 
batic cut-off of $\phi(x)$ (see Fig. 2). This cut-off determines also the lower limit of $W$ for given values of $b$ and $\omega>\omega_{t h}\left(\propto E_{t h}\right.$ ' the break up threshold).

Simultaneously one has to consider the elastic scattering, which may be an origin of experimental problems, when studying the break up reactions at very forward reaction angles. The elastic scattering cross section

$$
\frac{d \sigma^{\text {Ruth }}}{d \Omega}=\frac{a^{2}}{4 \sin ^{4}\left(\frac{\Theta}{2}\right)}=\frac{b^{\frac{4}{2}}}{4 a^{2}}=\frac{b^{4} W^{2}}{e^{4} z_{T}^{2}}\left(\frac{A}{z_{P}}\right)^{2}
$$

increases with $\mathrm{w}^{2}$ and with $\mathrm{b}^{4}$.

Therefore it is obvious that the impact parameter value should be chosen not larger than required by the condition of vanishing nuclear field.

For numerical estimates for the two examples under consideration $b=10 \mathrm{fm}$ is adopted, where presumably the influence of the nuclear field is negligible. The threshold $E_{t h}=1.586 \mathrm{MeV}$ for the ${ }^{7} \mathrm{Be} \rightarrow \alpha+{ }^{3} \mathrm{He}$ reaction corresponds to $\mathrm{W}_{\min }=3.04$, the ${ }^{16} \mathrm{O} \rightarrow{ }^{12} \mathrm{C}+\alpha$ threshold $\mathrm{E}_{\text {th }}=7.162 \mathrm{MeV}$ to $\mathrm{W}_{\mathrm{min}}=62$. We see, the larger threshold for the ${ }^{16} \mathrm{O}$ dissociation requires considerably higher projectile energies, with increased experimental difficulties as the pure Coulomb dissociation is restricted to a rather small angular range in extreme forward direction.

For the threshold photon energies the virtual photon numbers are explicitly given by

$$
\frac{\mathrm{dn}_{E 1}}{\mathrm{~d} \Omega}\left(\mathrm{b}=10 \mathrm{fm}, E_{\gamma}=1.586 \mathrm{MeV}, \mathrm{W}\right)=50.5 \mathrm{~W} \phi\left(\frac{1.74}{\sqrt{\mathrm{W}}}\right)
$$

for the ${ }^{7}$ Be break up and

$$
\frac{d n_{E 1}}{d \Omega}\left(b=10 \mathrm{fm}, E_{Y}=7.162 \mathrm{MeV}, W\right)=66 \mathrm{~W} \phi\left(\frac{7.86}{\sqrt{W}}\right)
$$

for the ${ }^{16}$ o break up.

The $W$-dependence is plotted in Fig. 4 and the virtual photon spectra are shown in Fig. 5 for different projectile energies for for a value of the impact parameter $b=10 \mathrm{fm}$ (see also Fig. 2). 


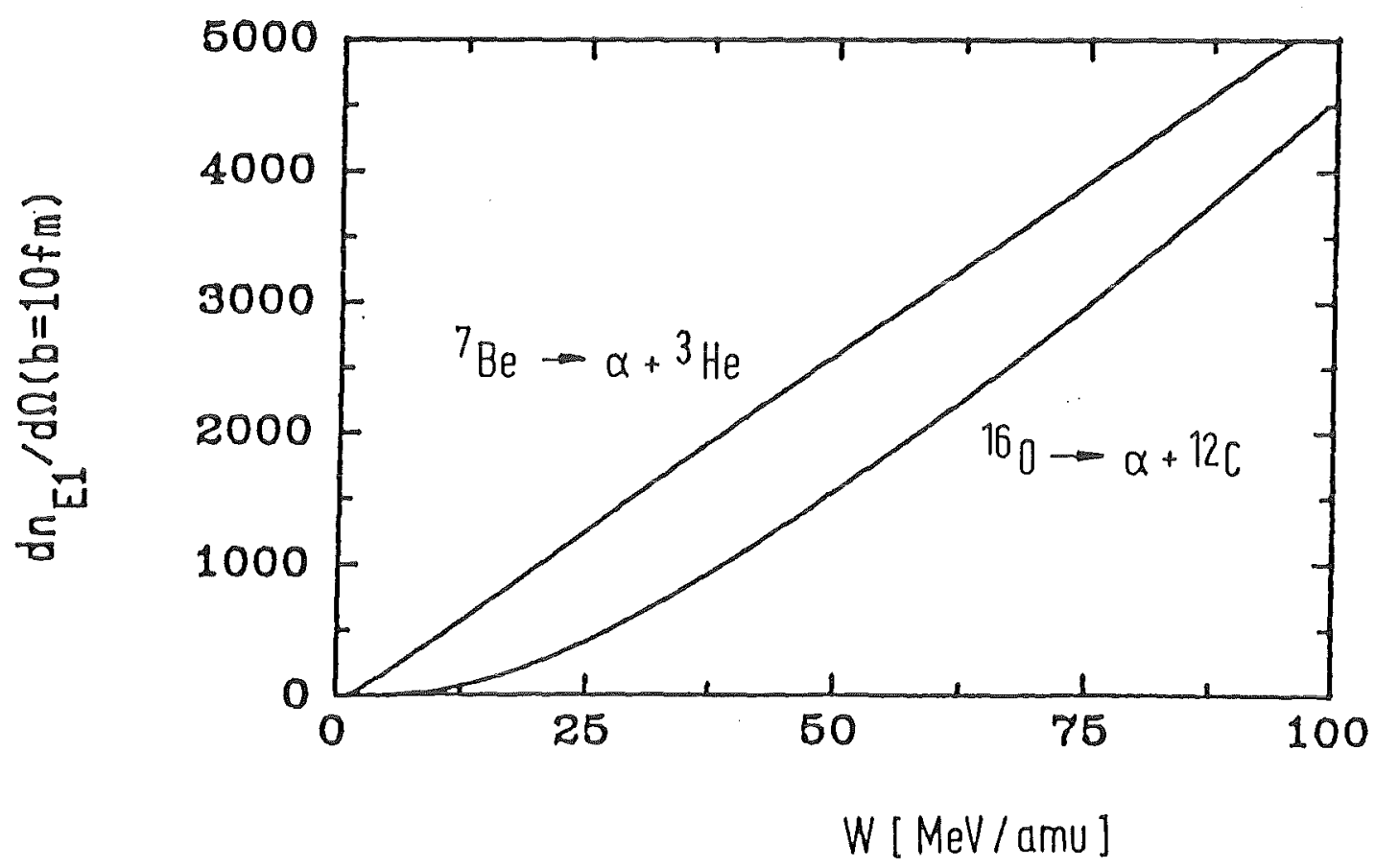

Fig. 4 Virtual photon numbers (E1-component) for the threshold energies of the two considered cases as function of the projectile energy.

In Fig. 6 for the ${ }^{16} 0$ dissociation at the threshold the total virtual photon number $\mathrm{n}_{\mathrm{E}}{ }$, integrated over all scattering angles (impact parameters) is displayed as calculated on the basis of the three different expansions representing the nonrelativistic (eq. 2.15) and the relativistic (eq. 2.16) limits and the intermediate energy region (eq. 2.14). The eq. 2.14 includes the necessary modifications when the parameter $\xi=x / \varepsilon=\omega a / v$ is appreciably larger than zero (see Fig. 3) and orbital dispersion and retardation effects show up (see sect. 2). The comparison of the different expressions shows the over-estimation of $n_{E 1}$ by the relativistic limit at lower energies and the underestimation by the nonrelativistic limit expression at higher projectile energies.

In Fig. 7 the total virtual photon number $\mathrm{n}_{\mathrm{E} 1}$ for the ${ }^{7} \mathrm{Be}+{ }^{208} \mathrm{~Pb} \rightarrow \alpha+{ }^{3} \mathrm{He}+{ }^{208} \mathrm{~Pb}$ dissociation is shown as function of the kinetic center of mass energy $\mathrm{E}_{\alpha-{ }^{3} \mathrm{He}}$ in. the system of the 


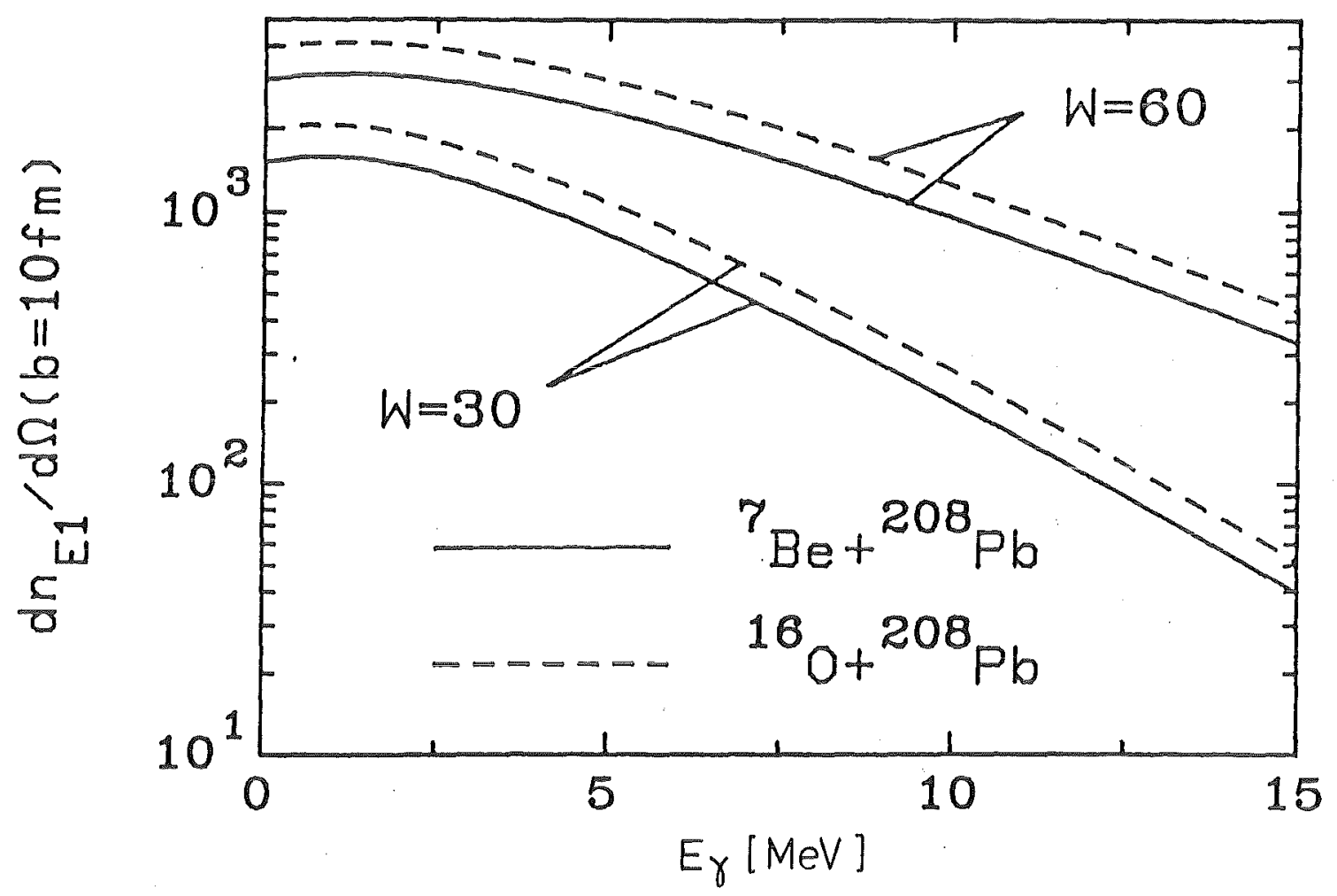

Fig. 5 E1 virtual photon spectra, seen by the projectiles with $\mathrm{b}=10 \mathrm{fm}$ at different projectile energies.

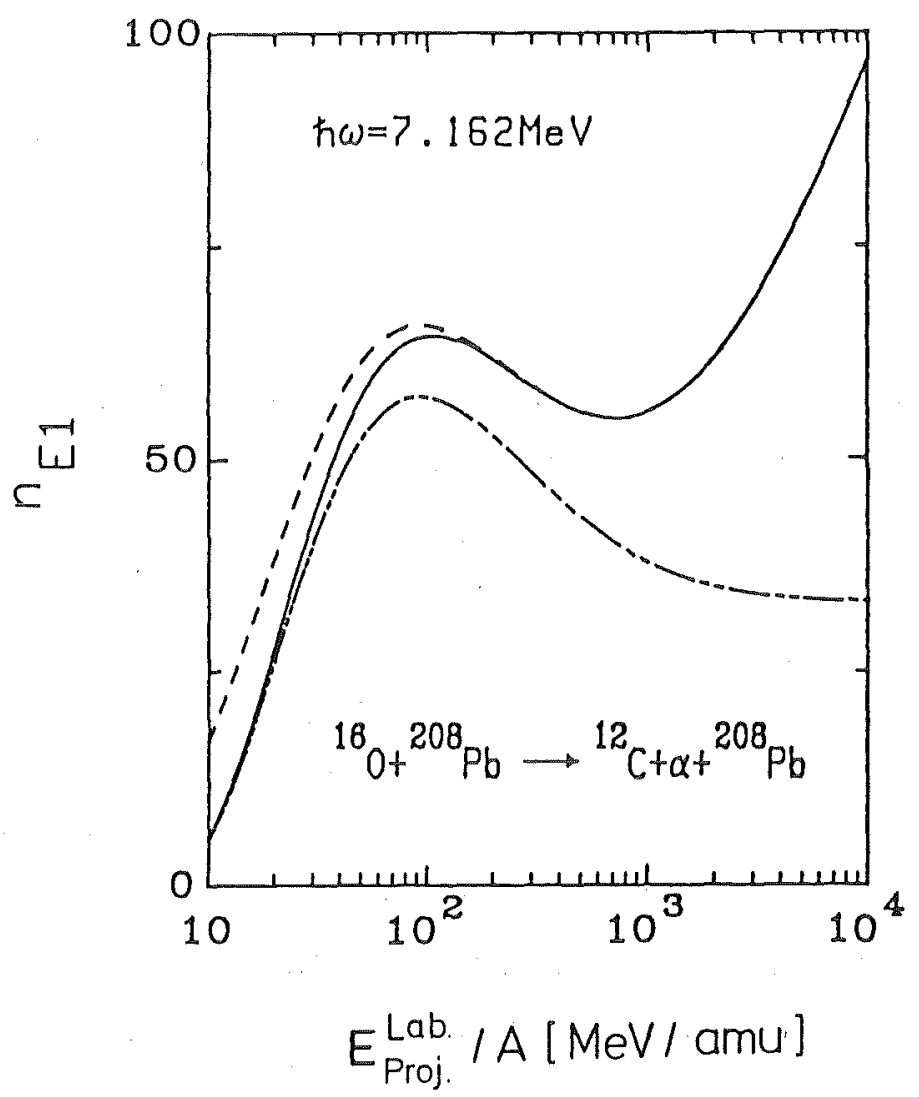

Fig. 6 The total virtual photon number $\left(E_{\gamma}=7.162 \mathrm{MeV}\right)$ as calculated for ${ }^{16} \mathrm{O}$ break up on the basis of the expressions eq. $2.14 \longmapsto$, eq.2.15(---) and eq. $2.16(---)$. 
emerging ${ }^{3} \mathrm{He}$ and $\alpha$-particles at various laboratory energies of the incident ${ }^{7}$ Be projectiles.

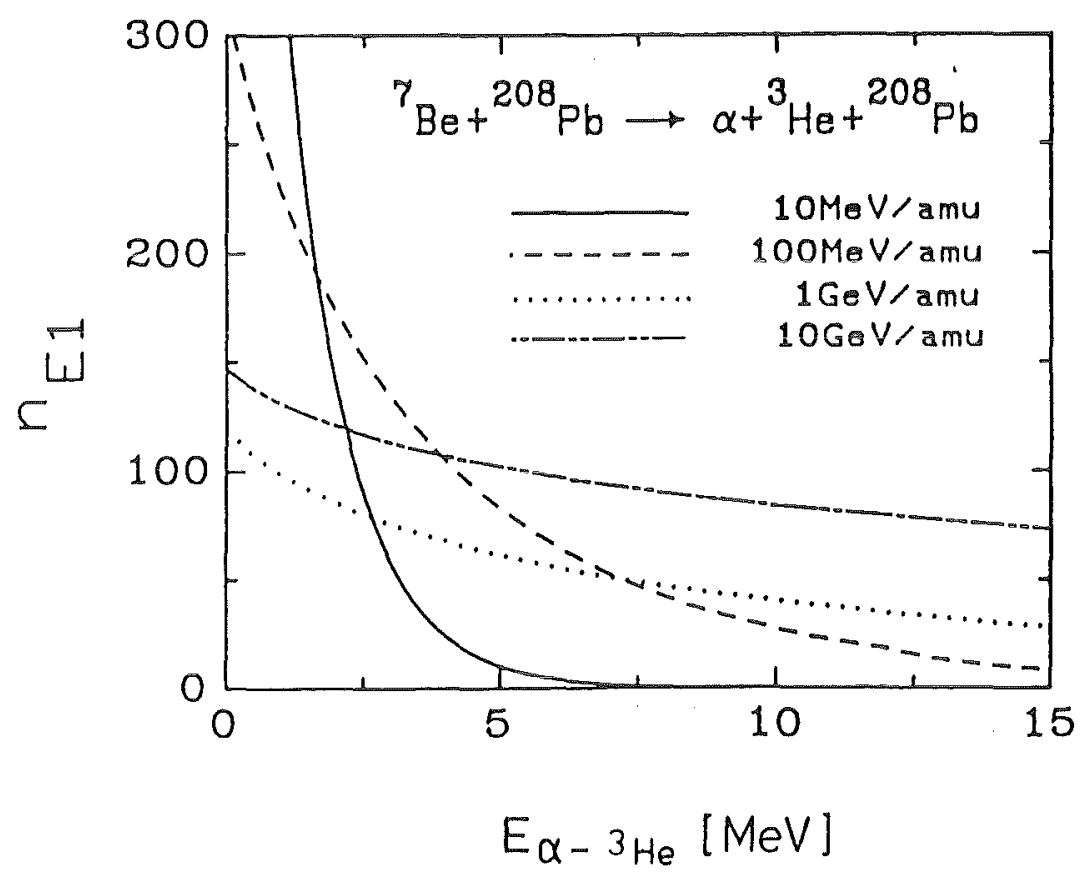

Fig. 7 Angle-integrated virtual photon spectra plotted for various 7 Be projectile energies as function of the kinetic center of mass energy of the emerging ${ }^{3} \mathrm{He}$ and $\alpha$-particles after Coulomb dissociation.

It indicates the favourable features of lower energy projectiles for dedicated studies of the break up with small relative energies in the system of the escaping fragments.

For an estimate of the cross section for the ${ }^{7} \mathrm{Be} \rightarrow \alpha+{ }^{3} \mathrm{He}$ break up we adopt the astrophysical $\mathrm{S}$-factor $S=0.5 \mathrm{keV} \cdot \mathrm{b}$ (ref. 5), which corresponds to a capture reaction cross section $\sigma_{\text {capt }}\left(E_{\alpha-{ }^{3} \mathrm{He}}\right.$ $=100 \mathrm{keV}) \simeq 0.5 \mathrm{nb}$ or a photodisintegration cross section $\sigma\left({ }^{7} \mathrm{Be}+\gamma \rightarrow \alpha+{ }^{3} \mathrm{He}, \mathrm{E}_{\gamma}=1.658 \mathrm{MeV}\right) \simeq 14 \mathrm{nb}$ (see eq. 1.4). The ${ }^{12} \mathrm{C}(\alpha, \gamma){ }^{16} \mathrm{O}$ cross section extrapolated to $\mathrm{E}_{\alpha-1{ }^{2} \mathrm{C}}=1 \mathrm{MeV}$ (see ref. 6) is of the order $\sigma_{\text {capt }} \approx^{10^{-1}} \mathrm{nb}$. The corresponding photointegration cross section $\sigma\left({ }^{16}{ }_{O+\gamma \rightarrow}{ }^{12} \mathrm{C}+\alpha, \mathrm{E}_{\gamma}=8.162 \mathrm{MeV}\right) \approx 3 \mathrm{nb}$ is enhanced by a factor of about 30. Applying eq. 2.3 with the results of sect. 2 for virtual photon number $\mathrm{dn}_{\mathrm{E} 1} / \mathrm{d} \Omega$ the values of the double-differ- 
ential cross section for exciting the projectile to an excitation energy $E_{X}=E_{\gamma}$ by the Coulomb field of a $P b$ nucleus, passed with an impact parameter $b\left(=z_{p} \cdot z_{T} e^{2} / 2 E_{p} \cdot \operatorname{ctg} \theta / 2\right)$ are estimated $\left(b=10 \mathrm{fm}, E_{p}=30 \mathrm{MeV} / \mathrm{amu}\right.$ )

$$
\frac{d^{2} \sigma}{d \Omega d E_{x}} \simeq 11 \mathrm{mb} \mathrm{MeV}^{-1} \text { sterad }^{-1}
$$

in the case of ${ }^{7} \mathrm{Be} \rightarrow \alpha+{ }^{3} \mathrm{He}$, and

$$
\frac{\mathrm{d}^{2} \sigma}{\mathrm{d} \Omega \mathrm{dE} \mathrm{E}_{\mathrm{x}}} \simeq 2 \mu \mathrm{bMeV}-1 \text { sterad }^{-1}
$$

in the case of ${ }^{16} \mathrm{O} \rightarrow{ }^{12} \mathrm{C}+\alpha$.

\section{Conditions of experimental investigations}

The emission of light particles is a quite usual feature in reactions of two complex nuclei and is associated with various different reaction mechanisms. The elastic projectile break up: $a \rightarrow b+c$ (leaving the target nucleus in the ground state) comprises only a minor part of the total reaction cross section. Detailed and unique information about the process under consideration requires inevitably kinematically complete experiments. The fragments resulting from a binary dissociation in flight of a nuclear projectile, after being scattered and excited to a particular value of the excitation energy $\mathrm{E}_{\mathrm{x}}$ (in the continuum) have limits imposed on the energies with which they appear in the laboratory. Their kinetic energies in their CM system seem to the relative energy $E_{b c}=E_{x}-Q$. The relevant momenta are illustrated in Fig.8. Here $\vec{p}_{O b}$ and $\vec{p}_{O C}$ stand for the fragments shares of the projectile momentum with

$$
\begin{aligned}
& \left|\overrightarrow{\mathrm{p}}_{\mathrm{Ob}}\right|=\sqrt{\frac{\mathrm{m}_{\mathrm{b}}^{2}}{\frac{\mathrm{m}_{\text {Proj }}}{\mathrm{E}_{\mathrm{O}}^{l a b}}}} \\
& \left|\overrightarrow{\mathrm{p}}_{\mathrm{OC}}\right|=\sqrt{\frac{\mathrm{m}_{\mathrm{c}}^{2}}{\mathrm{~m}_{\text {Proj }}} \mathrm{E}_{\mathrm{O}}^{l \mathrm{ab}}}
\end{aligned}
$$


The fragment laboratory momenta are resultants of their shares in the projectile and of $\overrightarrow{\mathrm{p}}_{\mathrm{bc}}=\mu_{\mathrm{bc}} / \mathrm{m}_{\mathrm{b}} \cdot \overrightarrow{\mathrm{p}}_{\mathrm{b}}+\mu_{\mathrm{bc}} / \mathrm{m}_{\mathrm{c}} \cdot \overrightarrow{\mathrm{p}}_{\mathrm{c}}$, the momentum they pick up from the internal energy $E_{b c}$ of the "projectile" after dissociation. Since $\left|\vec{p}_{b c}\right|=\sqrt{2 \mu_{b c} E_{b c}}$ is rather small in cases of our interest as compared with the momenta associated with the projectile motion, both fragments emerge in the laboratory at fairly small angles to the direction of the scattered "projectile" (direction of the $(b+c)$-subsystem).

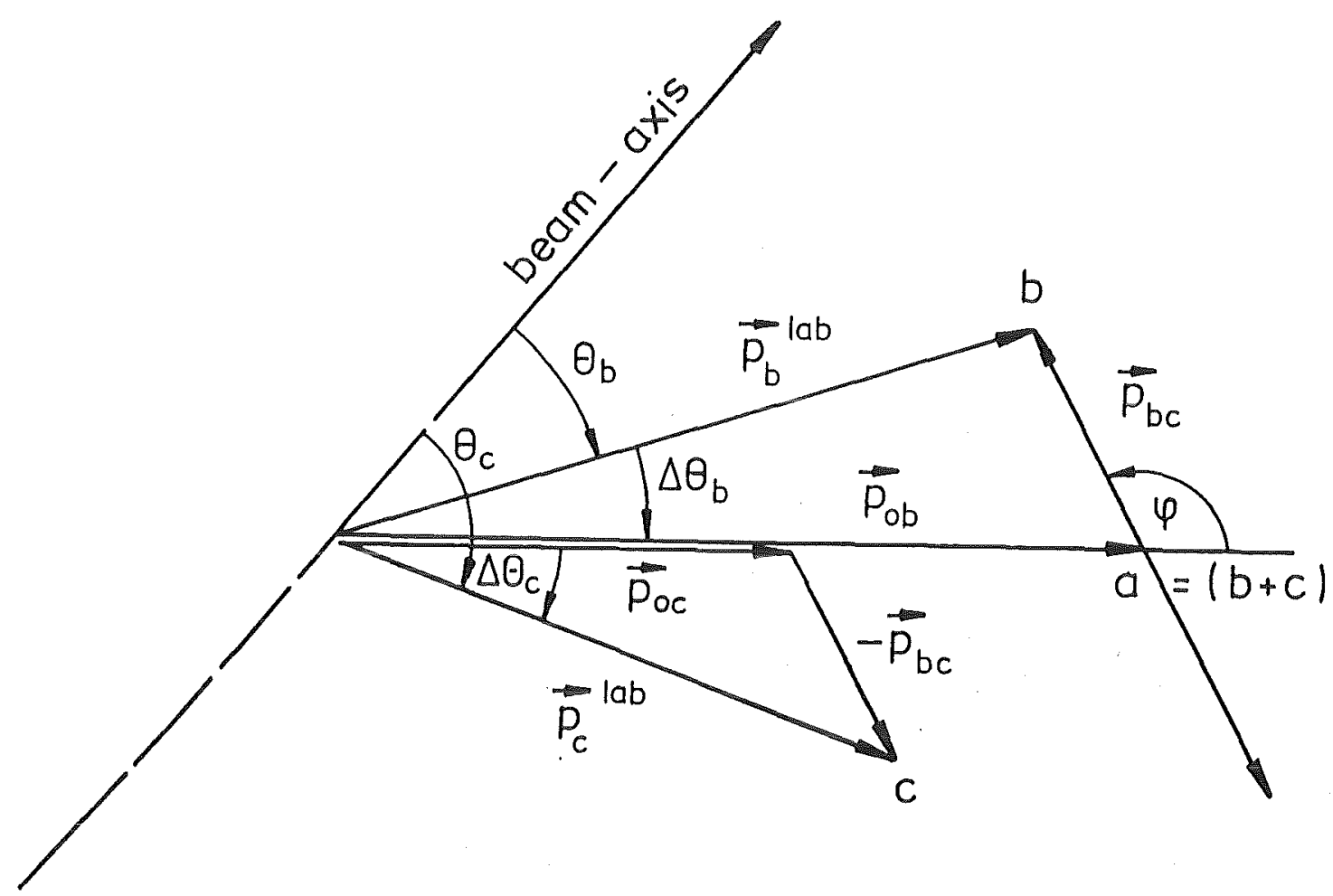

Fig. 8 Kinematics of the $a \rightarrow b+c$ break up

The laboratory energies and emission angles of the fragments are correlated by their dependence on the angle $\Phi$ at which the particles are emitted in their rest system

$$
E_{b}^{1 a b}=\frac{m_{b}}{m_{\operatorname{Proj}}} E_{o}^{1 a b}+\frac{\mu_{b c}}{m_{b}} E_{b c}+2 \sqrt{\frac{\mu_{b c}}{m_{\operatorname{Proj}}} E_{o}^{l a b} \cdot E_{b c}} \cos \Phi
$$




$$
\begin{aligned}
& E_{c}^{l a b}=\frac{m_{c}}{m_{\text {Proj }}} E_{o}^{l a b}+\frac{\mu_{b c}}{m_{c}} E_{b c}-2 \sqrt{\frac{\mu_{b c}}{m_{\text {Proj }}} E_{o}^{l a b} \cdot E_{b c}} \cos \Phi \\
& \sin \Delta \theta_{b}=\left|\vec{p}_{b c}\right| /\left|\vec{p}_{o b}\right| \cdot \sin \Phi=\sqrt{\frac{m_{P r o j} \cdot \mu_{b c}}{m_{b}^{2}} \frac{E_{b c}}{E_{o}^{l a b}}} \sin \Phi \\
& \sin \Delta \theta_{c}=\left|\vec{p}_{b c}\right| /\left|\vec{p}_{o c}\right| \cdot \sin \Phi=\sqrt{\frac{m_{P_{r o j}} \mu_{b c}}{m_{c}^{2}} \frac{E_{b c}}{E_{o}^{l a b}}} \sin \Phi
\end{aligned}
$$

The maximum opening angles (with respect to the direction of the scattered "projectile") for a given relative energy $\mathrm{E}_{\mathrm{bc}}$ are given by

$$
\begin{array}{ll}
\sin \Delta \theta_{b}^{\max } & \simeq \sqrt{\frac{m_{c}}{m_{b}} E_{b c} / E_{o}^{1 a b}} \\
\sin \Delta \theta_{c}^{\max } & \simeq \sqrt{\frac{m_{b}}{m_{c}} E_{b c} / E_{o}^{l a b}}
\end{array}
$$

As an example we consider the dissociation of ${ }^{7} \mathrm{Be} \rightarrow \alpha+{ }^{3} \mathrm{He}$ by Coulomb scattering on ${ }^{208} \mathrm{~Pb}$ with an incident energy of $\mathrm{E}_{\mathrm{LAB}}=$ $210 \mathrm{MeV}(\eta=9.4)$ at $\Theta_{\mathrm{Lab}}=6.4^{\circ}$ (corresponding to $\mathrm{b}=\mathrm{a} \mathrm{ctg} \theta_{\mathrm{CM}} / 2=$ $10 \mathrm{fm})$ and with the relative energy $\mathrm{E}_{\alpha-{ }^{3} \mathrm{He}}=0.1 \mathrm{MeV}$ of the emerging fragments. The laboratory energy of the scattered ${ }^{7} \mathrm{Be}$ (i.e. of the $\alpha^{-3} \mathrm{He}$ center of mass) $\mathrm{E}_{\mathrm{O}}^{\mathrm{lab}}=208.2 \mathrm{MeV}$ and it follows that the break up fragments of that particular value of $\mathrm{E}_{\alpha-{ }^{3} \mathrm{He}}$ are emitted within $\Delta \theta_{\alpha}^{\max }=1.1^{\circ}$ and $\Delta \Theta_{3} \mathrm{He}=1.5^{\circ}$ off the direction of the $\theta_{\text {Lab }}=6.4^{\circ}$. The extreme energies for a definite $E_{\alpha-3}$ He occur when the break up happens in line with the projectile flight directions, and $\mathrm{E}_{\alpha}^{\mathrm{lab}}$ (max) occurs together with $\mathrm{E}_{3}{ }_{\mathrm{He}}$ (min) and vice versa. The width of the energy window (roughly centered around the "beam velocity" energy) is

$$
\Delta E=4 \sqrt{\frac{\mu_{b c}}{m_{\text {Proj }}} E_{o}^{1 a b} E_{b c}}(=28.6 \mathrm{MeV} \text { for our case })
$$

Within this window a particular combination $\left(\mathrm{E}_{\mathrm{b}}^{\mathrm{lab}}, \mathrm{E}_{\mathrm{c}}^{\mathrm{lab}}\right)$ (eqs. $4.2 \mathrm{a}-\mathrm{b}$ ) with corresponding values $\left(\theta_{\mathrm{b}}, \theta_{\mathrm{C}}\right)$ (eqs. $4.3 \mathrm{a}-\mathrm{b}$ ) corresponds to a particular value of $\Phi$ for a specific value of $\mathrm{E}_{\mathrm{bc}}$. This kinematic feature enables, in principle, the access to information on the angular distribution of the dissociation. 
In a typical experimental situation two particle detector telescopes measure ${ }^{+}$the energies $E_{b}^{1 a b}$ and $E_{C}^{l a b}$ of the particles $b$ and $c$ emitted with fixed laboratory angles $\theta_{b}$ and $\theta_{c}$, say in a "in-plane" geometry, so that the sum of the opening angles $\Delta \theta_{b}+\Delta \theta_{c}$ is given. The coincidence events related to elastic break up of the projectile follow ${ }^{19}$ a well defined curve in the $\mathrm{E}_{\mathrm{b}}^{1 \mathrm{ab}}$ $\mathrm{E}_{\mathrm{b}}^{\mathrm{lab}}$ - plane. For a heavy target and a light projectile the kinematical curve is distorted to nearly a straight line. For the two considered cases and for a particular detector set up the kinematical loci (calculated on the basis of the relativistic kinematics) are shown in Fig. 9 and Fig.10. The allowed $E_{b}^{l a b}-E_{c}^{1 a b}$ combinations map the variation of the relative energy $\mathrm{E}_{\mathrm{bc}}$ " which varies remarkably slowly with the laboratory energies (see insets).

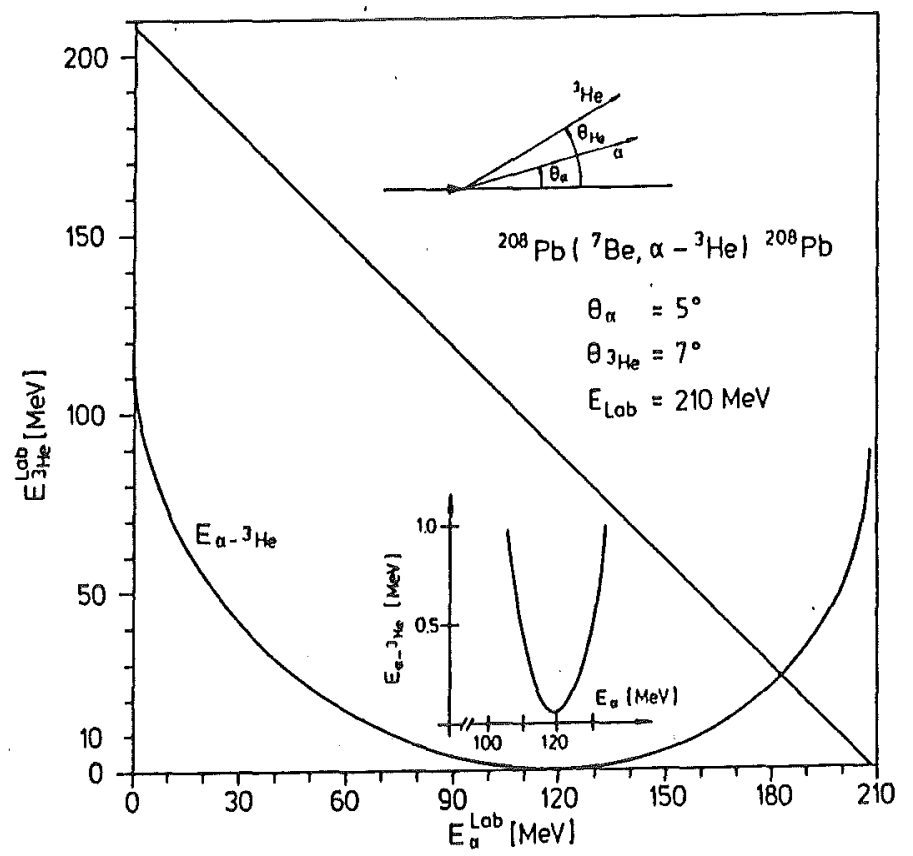

Fig. 9 Kinematical loci of the emerging ${ }^{3} \mathrm{He}$ and $\alpha$-particles From a ${ }^{7} \mathrm{Be}$ dissociation on ${ }^{208} \mathrm{~Pb}$ at $\mathrm{E}_{1 \mathrm{ab}}=30 \mathrm{MeV} / \mathrm{amu}$. The variation of $E_{\alpha-{ }^{3} \mathrm{He}}$ around the minimum $\left(E_{\alpha-3}^{\min } \mathrm{He}=63 \mathrm{keV}\right)$ is shown in the inset.

+ For sake of simplicity we assume the one detector observes only the particles $b$, the other only the particles $c$. 
This "magnifying glass" effect enables detailed studies of the variation of the cross section with $E_{b c}$. However, one has to realize that the emission angle $\Phi$ and the partition $\left(\Delta \theta_{b}, \Delta \theta_{c}\right)$ for the given $\left(\Delta \Theta_{b}+\Delta \Theta_{c}\right)$ value changes, too. In fact, there are two branches of $\mathrm{E}_{\mathrm{bc}}$, in general corresponding to different emission angles $\Phi$ in the rest system ${ }^{20}$ and toslightly different directions of the $(b+c)$ $C M$ motion for a specific value of $E_{b c}$ on both branches. The minimum of $\mathrm{E}_{\mathrm{bc}}$ is determined by the angular spacing of the two telescopes.

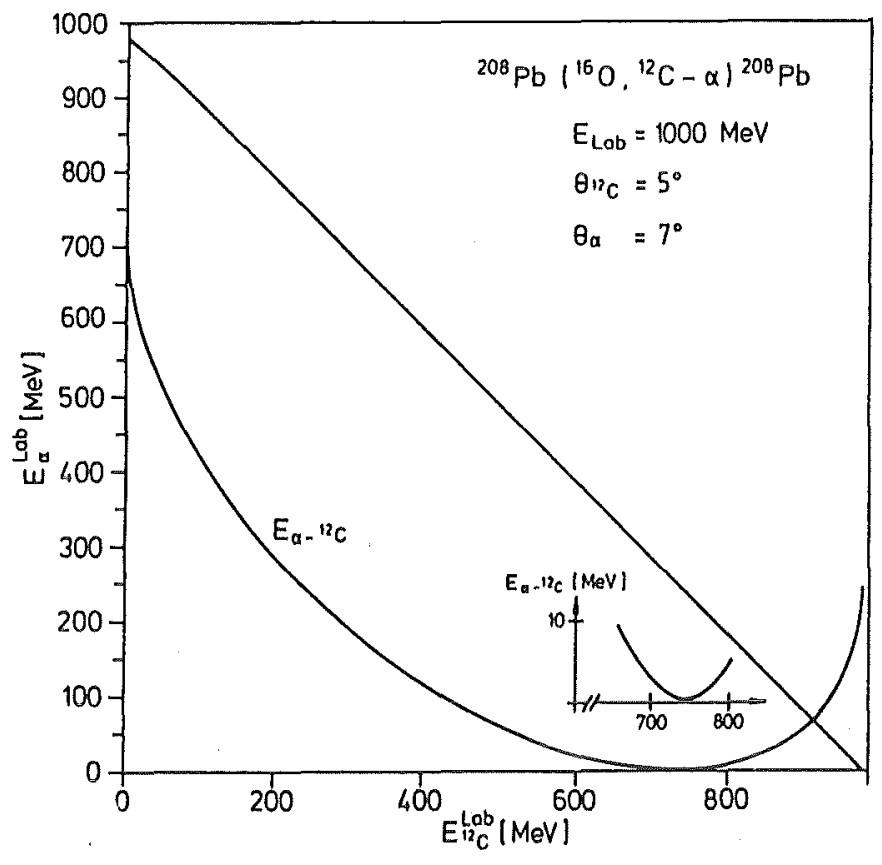

Fig. 10 Kinematical loci of the emerging $\alpha$-particles and ${ }^{12} \mathrm{C}$ from the ${ }^{16} \mathrm{O}$ dissociation on ${ }^{208} \mathrm{~Pb}$ at $\mathrm{E}_{\mathrm{Lab}}=30 \mathrm{MeV} / \mathrm{amu}$ $\left(E_{\alpha-12 C}^{\min }=235 \mathrm{keV}\right)$ 
The flexibility of the kinematics is an inherent advantage of the approach discussed here.

For an estimate of the triple differential laboratory cross section $d^{3} \sigma / d E_{b} d \Omega_{b} d \Omega_{c}$ we assume, for sake of simplicity, isotropic emission of the fragments $b$ and $c$ in their rest system. Then

$$
\frac{d^{3} \sigma}{d E_{b c}{ }^{d} \Omega_{b c}{ }^{d} \Omega_{\text {Target }-(b+c)}}=\frac{1}{4 \pi} \quad \frac{d^{2} \sigma}{d E_{b c}{ }^{d \Omega} \text { Target }-(b-c)}
$$

where $d E_{\mathrm{bc}} \equiv \mathrm{dE} \mathrm{x}_{\mathrm{x}}$ and $\mathrm{d} \Omega_{\mathrm{Target}-(\mathrm{b}+\mathrm{c})} \equiv \mathrm{d} \Omega$ in the expressions of sect. 3. Evaluating the transformation from the relative and center of mass motion of the two fragments to the laboratory system (using the expressions of ref. 21) for the ${ }^{208} \mathrm{~Pb}\left({ }^{7} \mathrm{Be}, \alpha-{ }^{3} \mathrm{He}\right){ }^{208} \mathrm{~Pb}_{\mathrm{g}}{ }^{-}$ example in the situation of $\mathrm{Fig} .9$, we find for $\mathrm{E}_{\alpha^{3} \mathrm{He}}=0.1 \mathrm{MeV}$ (at $\mathrm{E}_{\alpha}^{\mathrm{lab}}=116 \mathrm{MeV}$ and $\mathrm{E}_{3}^{\mathrm{lab}} \mathrm{He}=92.3 \mathrm{MeV}-$ see Fig. 9)

$$
\frac{d^{3} \sigma}{d E_{\alpha} d \Omega_{\alpha} d \Omega_{3} \mathrm{He}} \approx 52 \mu \mathrm{b} \cdot \mathrm{MeV}^{-1} \text { sterad }^{-2} \text {. }
$$

This value appears to be measurable by special experimental efforts. One of the main difficulties arises from the dominant competition of the elastic scattering in forward direction with a differential cross section ca. 6 orders of magnitude larger. When detecting the break up fragments with magnetic spectrometers, it is possible to suppress strongly the elastic scattering by an effective shadowing ${ }^{22}$ of the corresponding position in the focal plane, thus drastically reducing accidental coincidences and admitting larger beam currents.

Very interesting and improved experimental possibilities would be provided by a dedicated set up at a synchotron-cooler ring (see ref. 23) with suitable magnetic spectrometers (like the proposal of ref. 24) enabling particle coincidence studies at very forward emission directions. The use of a storage ring seems to be indispensable when working with radioactive beams like with ${ }^{7}$ Be. Even, if the acceleration and preparation of such a beam would be successful in a conventional approach, the contamination problems arising from the accumulation of the radioactivity $\left(\mathrm{T}_{1 / 2}\left[{ }^{7} \mathrm{Be}\right]=\right.$ 53.3 d) impose serious limits. On the other side, in a storage ring a current of $100 \mathrm{~mA}$ corresponds to a sufficiently small 
number of stored radioactive particles. A Hg vapour jet target 25 e.g. may serve as reaction target for the Coulomb break up measurements.

\section{Conclusions}

The proposed approach for studies of the interaction of nuclear particles at small relative energies requires experiments at extreme forward angles, in a region where $\sigma_{\text {elastic }} / \sigma_{R}=1$. The elastic scattering cross section provides, in fact, a calibration of the break up cross sections. The values of the estimated coincidence cross sections are rather small, but appear to be measurable by present days" experimental techniques. The kinematic situation with three outgoing particles provides particular advantages for studies of the excitation function i.e. the variation with relative energy of the emerging fragments, and of the angular distribution in the rest frame of the fragments subsystem. Investigation of the latter aspect, however require a quite good angular resolution. The cross sections can be interpreted in terms of electromagnetic interaction matrix elements which just determine the radiative capture cross section. By considering particular cases of astrophysical interest we have demonstrated that we can extend the information to rather low relative energy presently not accessible for direct capture measurements. With the same conclusion, a further example, the coulomb break up of ${ }^{6} \mathrm{Li}$ at low $(\alpha+d)$ relative energies has been recently analysed $^{26}$ on the basis of a DWBA approach which includes distortion effects.

We acknowledge valuable discussions with H.J. Gils, L. Lassen, G. Schatz and D.K. Srivastava. 


\section{References}

1 W.A. Fowler, Rev. Mod. Phys. 56 (1984) 149

2 C. Rolfs and H.P. Trautvetter, Ann. Rev. Nucl. Sci. 28 (1978) 115

3 Toshitaka Kajino and Akito Arima, Phys. Rev. Lett. $\underline{5} 2$ (1984) 739

4 J.L. Osborne, C.A. Barnes, R.W. Kavanagh, R.M. Kremer, G.J. Mathews, J.L. Zyskind, P.D. Parker, and A.J. Howard, Phys. Rev. Lett. $\underline{4} 8$ (1982) 1664 -

Nuc1. Phys. A419 (1984) 115

5 K. Nagatani, M.R. Dwarakanath, and D. Ashery, Nucl. Phys. A128 (1969) 325

6 K.U. Kettner, H.W. Becker, L. Buchmann, J. Görres, H. Kräwinkel, C. Rolfs, P. Schmalbrock, H.P. Trautvetter, and A. Vlieks Z. Phys. A308 (1982) 73

K. Langanke and S.E. Koonin, Nucl. Phys. A439 (1985) 384

7 G. Baur, B. Hoffmann, F. Rösel, D. Trautmann, and R. Shyam, Proc. Workshop COPECOS, Bad Honnef, 1984 (World Scientific Publ. Comp., Singapore, 1984) p. 538;

G. Baur, F. Rösel, D. Trautmann, and R. Shyam, contribution to the 6th Int. Symp. on Polarization Phenomena in Nuclear Physics, Osaka 1985, p. 105

8 H. Rebel, Workshop "Nuclear Reaction Cross Sections of Astrophysical Interest", unpublished report, Kernforschungszentrum Karlsruhe, February 1985

9 C.A. Bertulani and G. Baur, Nucl. Phys. A 4442 (1985) 739

$10 \mathrm{~K}$. Alder and A. Winther, Electromagnetic Excitation (North-Holland, Amsterdam. 1975)

K. Alder and A. Winther, Coulomb Excitation (Academic Press, New York 1966)

11 H.A. Weidenmüller and A. Winther, Ann. Phys. 66 (1971) 218

12 B. Hoffmann and G. Baur, Phys. Rev. 30 (1984) 247

13 K. Alder, A. Bohr, T. Huus, B. Mottelson, and A. Winther, Rev. Mod. Phys. 2ㅇ (1956) 432, and references therein. 
14 K.A. Ter-Martirosyan, Zh. Eksperim.i. Theor. Fiz. 22 (1952) 284. Reprinted in Ref. 10

15 E. Fermi, Z. Phys. 29 (1924) 315

16 C.F. Weizsäcker, Z. Phys. $8 \underline{8}$ (1934), 612

E.J.Williams, Phys. Rev. $4 \underline{5}$ (1934) 729

17 J.D. Jackson, Classical Electrodynamics (Wiley, New York (1975) p. 719

18 G.N. Watson, Theory of Bessel functions (Cambridge University Press, New York, 1958) second edition, p. 133.

19 G.G. OhIsen, Nucl. Instr. and Meth. 37 (1965) 240

20 D. Scholz, H. Gemmeke, L. Lassen, R. Ost, and K. Bethge Nuc1. Phys. A288 (1977) 351

C.M. Castaneda, H.A. Smith Jr., P.P. Singh, and H. Karwowski Phys. Rev. C21 (1980) 179

21 H. Fuchs, Nucl. Instr. and Meth. 200 (1982) 361

22 H.J. Gils, H. Jelitto, S. Zagromski, W. Eyrich and H. Schlösser, in Annual Report on Nuclear Physics Activities (KfK 3969:-1985, eds. K. Bekk, P. Doll and Ch. Weddigen) Contr. 1.3.11, p.47

23 COSY, Proposal for a cooler-synchotron as a facility for nuclear and intermediate energy physics at the KFA Jülich, Jülich 1985; Studie zum Bau eines kombinierten Kühler-Synchotron Rings an der KFA Jülich - Jül-Spez-242 Februar 1984 ISSB $0343-7639$

24 J.W. Sunier, K.D. Bol, M.R. Clover, R.M. De Vries, N.J. Di Giacomo, J.S. Kapustinsky, P.L. MCGaughey and W.E. Sondheim, Nucl. Instr. and Meth. A241 (1985) 139

25 G. Montagnoli, H. Morinaga, U. Ratzinger and P. Rostek, Jahresbericht 1984 des Beschleunigerlaboratoriums der Universität und Technischen Universität München, contr.4.3.2. p. 109

26 D.K. Srivastava and H. Rebe1, Journal Phys. G. Nuc1. Phys. in press. 